\title{
The Impact of Cervical Stretching Exercise and Cervical Traction on Cervical Pain and Muscle Activity among Patients with Cervical Hypolordosis
}

\begin{abstract}
The purpose of this study is to provide fundamental clinical data for the treatment plan and rehabilitation of patients with cervical hypolordosis by comparing the cervical headache and muscle activity after cervical stretching exercise and cervical traction, which are generally applied to patients with cervical hypolordosis.

The research subjects included 20 patients without gender division who were diagnosed with cervical hypolordosis. After applying cervical stretching exercise and cervical traction for six weeks, cervical headache and the activity of the muscles around the cervical vertebra(upper trapezius muscle, sternocleidomastoid muscle, splenius capitis muscle, and anterior temporal muscle) were investigated and the following results were obtained. In a comparison of the within group intervention effects of the two groups, cervical pain statistically significantly decreased in the cervical stretching exercise group. According to the results of analyzing the change of muscle tension in the upper trapezius muscle, both the cervical traction group and showed a statistically significant within group difference in the left and right side. According to the results of analyzing the change in the muscle tension of the splenius capitis muscle, both groups showed a statistically significant within group difference in the left and right side. In a betweengroup comparison, a statistically significant difference in the right side was observed. These results confirm that cervical vertebra traction and cervical stretching exercise decrease the cervical headache and muscle activity of the upper trapezius muscle and the splenius capitis muscle among patients with cervical hypolordosis.
\end{abstract}

Key words: Cervical Stretching Exercise; Cervical Traction; Cervical Hypolordosis

\author{
Ho Jung $A n^{a}$, Jung Hyun Choi \\ ${ }^{a}$ Dongnam Health University, Suwon; ${ }^{\text {Namseoul }}$ \\ University, Cheonan, Korea \\ Received : 5 May 2015 \\ Revised : 15 June 2015 \\ Accepted : 20 August 2015 \\ Address for correspondence \\ Jung Hyun Choi, PT. Ph.D \\ Department of Physical Therapy, \\ Namseoul University, 21 Maeju-ri, \\ Sunghwan-eup, Cheonan, Korea \\ Tel: 82-41-580-2534 \\ E-mail: rightmind@nsu.ac.kr
}

\section{INTRODUCTION}

The head constitutes approximately $14 \%$ of human body weight, and the effective functionality of the neck and shoulder muscles is required to maintain correct stable posture(1). There are a number of patients with forward displacement of the head in our society these days due to inappropriate posture. Forward head posture increases the lordosis of the upper cervical vertebra and flattens the lower cervical vertebra. As this posture changes the balance on the neck, it causes muscle imbalance, tension in the cervical extension muscle, and the weakening of the deep cervical flexor muscle(1,2). As maintaining normal spine posture in a sitting posture becomes difficult, people tend to use a forward head posture where the head comes ahead of the center line of the spine. This posture induces the increase of lordosis in the cervical vertebra and the increase of kyphosis in the thoracic vertebrae and lumbar region. Due to the increase of kyphosis in the thoracic vertebrae, a decrease of vital capacity and thoracic cavity are caused in addition to round shoulder. In this case, pain, in particular, usually develops locally and is within $2 \%$ of spinal pain(3).

Neck pain is a frequently observed condition that approximately $70 \%$ of the total population experience at least once in their lifetime(1). When cervical pain becomes chronic, it can cause serious 
problems in the daily life of the patients. Many patients complain about headache, dizziness, and pain in the shoulder, arm, or between the shoulder blades, and these symptoms are directly and indirectly related to the problems in the cervical vertebra(3). Cervical pain can develop because of sudden injury, such as falling, sports injuries, and car accidents, and it can be aggravated due to the factors of inappropriate posture or continued working environment(4). Moreover, cervical vertebra injury or motor function anomalies can induce symptoms such as an avoidant defensive posture of the body, abnormal head tilt, and compensatory shoulder lift. The limited range of mobility of the cervical vertebra and facial asymmetry due to acute injury or chronic compensation can cause muscle convulsions around the spine and deterioration of muscle activity(5). When the head is tilted, the structural distortion and functional anomaly of the overall body are highly likely to occur due to the incongruity of the nerve system(3).

Cervical hypolordosis where the cervical vertebra decreases is a frequently observed finding among patients who visit orthopedics. Lee reported that $33.8 \%$ of the patients who visited the hospital because of cervical pain showed cervical hypolordosis(6). Comparing patients with cervical pain who received cervical stretching exercise and those who did not receive the stretching exercise, the group that underwent the stretching exercise showed not only an increase in muscle strength, but also a decrease in pain(7).

The cervical traction method is used for treating the cervical vertebra and the soft tissue around it that has been anatomically or mechanically transformed or deflected(8). Cervical traction is often used to ameliorate pain and increase mobility. When the traction method is applied to the cervical vertebra, articular surface separation and pressure decrease in the joint that has a diminished range of mobility. As a result, the movement of the articular surface increases, influencing the joint's mobility $(8,9)$.

The purpose of this study is to provide fundamental clinical data for the treatment plan and rehabilitation of patients with cervical hypolordosis by comparing the cervical headache and muscle activity after the cervical stretching exercise and cervical traction that are generally applied to patients with cervical hypolordosis.

\section{METHODS}

\section{Subjects}

For the subjects of this study, a total of 20 patients who reported discomfort and were diagnosed with cervical hypolordosis were chosen. The sample was divided into a cervical traction group(CTG) and a cervical stretching exercise group(CSEG). The inclusion criteria were as follows:

1) Patients without orthopedic defects (other than cervical hypolordosis transformation) that could affect the study results

2) Patients without defects in the central nerve system that could affect the study results

3) Patients without high blood pressure and coronary artery disease

4) Patients without innate type transformation

5) Patients without impairment in the vestibular system

Table 1. General features of research subjects

\begin{tabular}{ccc}
\hline & $\begin{array}{c}\text { Cervical traction } \\
\text { group } \\
\text { (Mean } \pm \text { SD) }\end{array}$ & $\begin{array}{c}\text { Cervical stretching } \\
\text { exercise group } \\
\text { (Mean } \pm \text { SD) }\end{array}$ \\
\hline Age $(\mathrm{y})$ & $43.53 \pm 5.41$ & $41.01 \pm 3.21$ \\
Height $(\mathrm{cm})$ & $162.07 \pm 4.42$ & $163.54 \pm 5.01$ \\
Weight $(\mathrm{kg})$ & $63.36 \pm 3.83$ & $67.14 \pm 5.21$ \\
\hline
\end{tabular}

All subjects voluntarily agreed to participate in the experiment. After sufficiently explaining the purpose and the implementation tasks of this study to all of the subjects, the study proceeded by obtaining participation agreements. The purpose of this study was accurately explained to all of the subjects and the subjects promised that they would not perform any additional treatment or exercise during the research period that could affect the study results.

\section{Measurement Method}

As for the tool for measuring the severity of cervical pain in this study, the visual analogue scale(VAS) was chosen among the pain evaluation indices that are mainly used in clinics. VAS was used as a means of investigating the pain change before and after the treatment by examining the severity of the oppressive pain of the neck muscles and headache among the research subjects. 
After drawing a $10 \mathrm{~mm}$ long line where the left end refers to a condition of no pain and the right end refers to maximal pain, the subjects were told to mark the severity of the pain in the neck muscle that they currently felt(10). The score was measured by checking the numbers at the marked places; a higher score meant more severe pain.

The activity of the muscles around the cervical vertebra was measured using a wireless electrode EMG system(Free EMG, BTS, Italy). Among the muscles around the cervical vertebra, the upper trapezius muscle, sternocleidomastoid muscle, splenius capitis muscle, and anterior temporal muscle were chosen, each of which were divided into the left and right side to yield a total of eight parts to be measured. To select the identical location for all subjects, the distance between the attachment location and the anatomical mark of the body was measured. To reduce the noise of the EMG measuring device and electrode cable, the cable was firmly attached using tape so that the electrodes would not detach from the subject during the experiment. To normalize the comparison of the EMG measurements among the subjects, the maximal voluntary isometric contraction (MVIC) of each subject was measured prior to the experiment. Data on each muscle in a condition of maximal strength against the isometric resistance for three seconds were collected(11). The MVIC values of each of the left and right side of the upper trapezius muscle, sternocleidomastoid muscle, splenius capitis muscle, and anterior temporal muscle were measured before the experiment.

The subjects took a stable posture and maintained each posture for one minute, during which time muscle loading was measured using EMG. Adding four minutes, muscle fatigue was measured over a total of five minutes. Fatigue effects were minimized by providing a five minute resting time between switching the postures. Muscle tension on the cervical erector spinae was diminished because the subjects lay down on a mat during the resting time. Moreover, measurement was conducted one by one in a lab for a single blind test(12).

\section{Cervical stretching exercise method and cervical traction method}

The cervical traction group and the cervical stretching exercise group performed the exercise three times a week for a total of six weeks. The implementation method is described below.

While maintaining a comfortable position, the cervical traction group took a supine position and intermittent traction consisting of 10 seconds of traction and 10 seconds of resting was applied for 20 minutes for the purpose of extending the rear cervical vertebra in a condition where the neck was in 20-30 degrees of flexion, the rope traction angle was approximately 60 degrees from the horizon, and the traction power was a weight of 8-14 kg(8,9).

Referring to the study by Lee, the cervical stretching exercise group maintained correct head neck posture in a group sitting position. Then, the participants did ascending and descending exercise of the shoulder joint, protraction and retraction exercise, horizontal adduction and abduction exercise along with the addition of bending to the left and the right, flexion, extension, and clockwise and counterclockwise rotation of the neck bone as voluntary exercises, with each exercise performed for 10 seconds followed by three seconds of resting time. Each set was repeated five times and performed for a total of three times(13). If a subject felt pain in a particular posture during the exercise, extension was performed within the range of no pain and then the exercise continued by gradually increasing the extension(Table 2 ).

Table 2 Stretching exercise program

\begin{tabular}{lll}
\hline Exercise & \multicolumn{1}{c}{ Method } & Frequency \\
\hline & $*$ position: sitting & \\
& $*$ holding time: $10 / \mathrm{sec}$ & \\
& $*$ rest: $3 / \mathrm{sec}$ \\
& $*$ repetition: 5 times & \\
& $*$ set: 3 times & \\
Stretching & - head and neck posture control & \\
exercise & - shoulder horizontal & \\
& - adduction elevation \\
& - shoulder depression \\
& - shoulder protraction \\
& - shoulder retraction \\
& - neck flexion/extension \\
& - neck side bending (Rt/Lt) \\
& - neck rotation (Rt/Lt)
\end{tabular}




\section{Data Analysis}

The data measured in this study were analyzed using SPSS ver. 18.0 software. General characteristics were expressed in means and standard deviations. Levene's F-test was used to test the homogeneity of the subjects and a KolmogorovSmirnov test was used to test normal distribution. A two way repeated measures ANOVA was con- ducted to compare the differences in the cervical pain and neck muscle activity variables according to group(cervical stretching exercise group and cervical traction group) and time (before and after the intervention). A paired t-test was used to test for significant differences between the pretest and posttest. A statistically significant level was set at $\alpha=.05$.

Table 3. Comparison of VAS by Cervical Pain

\begin{tabular}{|c|c|c|c|c|c|c|c|}
\hline Contents & Group & Pre(M $\pm S D)$ & Post(M $\pm S D)$ & $t$ & $p$ & $F$ & $p$ \\
\hline \multirow{2}{*}{ Pain } & CTG & $6.23 \pm .973$ & $5.32 \pm 1.871$ & 2.448 & .058 & \multirow{2}{*}{1.870} & \multirow{2}{*}{.201} \\
\hline & CSEG & $6.45 \pm .717$ & $.01 \pm 1.142$ & 2.741 & $.043^{*}$ & & \\
\hline
\end{tabular}

* p〈.05, CTG: cervical traction group, CSEG: cervical stretching exercise group

Comparing the within group intervention effects of the two groups, cervical pain statistically significantly decreased in CSEG ( $p<.05)$. A between- group comparison showed no statistically significant change in both oppressive pain and headache (p〉.05) (Table 3).

Table 4. Comparison of neck muscle activity. muscle activity(\% RVC).

\begin{tabular}{|c|c|c|c|c|c|c|c|c|}
\hline Cor & tents & Group & $\operatorname{Pre}(M \pm S D)$ & Post(M $\pm S D)$ & $t$ & $p$ & $\mathrm{~F}$ & $p$ \\
\hline \multirow{4}{*}{$\begin{array}{l}\text { Upper } \\
\text { trapezius } \\
\text { muscle }\end{array}$} & \multirow{2}{*}{$\mathrm{Rt}$} & CTG & $4.232 \pm 2.134$ & $3.814 \pm 1.047$ & 2.238 & .061 & \multirow{2}{*}{1.658} & \multirow{2}{*}{.20} \\
\hline & & CSEG & $4.321 \pm 3.214$ & $3.794 \pm 2.047$ & 2.454 & .054 & & \\
\hline & \multirow{2}{*}{$\mathrm{Lt}$} & CTG & $4.108 \pm 1.024$ & $3.940 \pm 1.247$ & 2.651 & $.047^{*}$ & \multirow{2}{*}{2.558} & \multirow{2}{*}{.111} \\
\hline & & CSEG & $4.214 \pm 2.147$ & $3.878 \pm 2.071$ & 2.689 & $.045^{*}$ & & \\
\hline \multirow{4}{*}{$\begin{array}{c}\text { Sternoclei } \\
\text { domas- } \\
\text { toid mus- } \\
\text { cle }\end{array}$} & \multirow{2}{*}{ Rt } & CTG & $6.321 \pm 2.321$ & $6.014 \pm 2.547$ & 1.950 & .113 & \multirow{2}{*}{.032} & \multirow{2}{*}{$.96 \varsigma$} \\
\hline & & CSEG & $6.874 \pm 3.014$ & $6.321 \pm 3.056$ & 1.701 & .149 & & \\
\hline & \multirow{2}{*}{$\mathrm{Lt}$} & CTG & $6.514 \pm 3.074$ & $5.957 \pm 3.914$ & 1.670 & .155 & \multirow{2}{*}{2.398} & \multirow{2}{*}{.125} \\
\hline & & CSEG & $6.214 \pm 2.248$ & $6.037 \pm 2.921$ & 1.784 & .137 & & \\
\hline \multirow{4}{*}{$\begin{array}{l}\text { Splenius } \\
\text { capitis } \\
\text { muscle }\end{array}$} & \multirow[t]{2}{*}{ Rt } & CTG & $39.841 \pm 13.587$ & $33.214 \pm 14.148$ & 2.587 & $.048^{*}$ & \multirow[t]{2}{*}{6.427} & \multirow[t]{2}{*}{.010} \\
\hline & & CSEG & $38.214 \pm 15.214$ & $35.321 \pm 17.321$ & 3067 & $.006^{*}$ & & \\
\hline & \multirow{2}{*}{$\mathrm{Lt}$} & CTG & $37.247 \pm 14.014$ & $34.047 \pm 16.584$ & 2.665 & $.037^{*}$ & \multirow{2}{*}{1.122} & \multirow{2}{*}{.351} \\
\hline & & CSEG & $39.841 \pm 13.587$ & $34.514 \pm 13.147$ & 2.954 & $.024^{*}$ & & \\
\hline \multirow{4}{*}{$\begin{array}{c}\text { Anterior } \\
\text { temporal } \\
\text { muscle }\end{array}$} & \multirow{2}{*}{ Rt } & CTG & $10.214 \pm 3.014$ & $9.514 \pm 2.957$ & .321 & .764 & \multirow{2}{*}{2.740} & \multirow{2}{*}{.097} \\
\hline & & CSEG & $11.574 \pm 4.142$ & $10.847 \pm 3.984$ & .264 & .801 & & \\
\hline & \multirow{2}{*}{$\mathrm{Lt}$} & CTG & $9.814 \pm 3.574$ & $9.114 \pm 4.257$ & .736 & .495 & \multirow[t]{2}{*}{.002} & \multirow[t]{2}{*}{.998} \\
\hline & & CSEG & $10.140 \pm 3.847$ & $9.847 \pm 4.084$ & .206 & .837 & & \\
\hline
\end{tabular}

p〈.05, CTG: cervical traction group, CSEG: cervical stretching exercise group 
Examining the muscle tension change in the upper trapezius muscle, a statistically significant within group difference was observed in both CTG and CSEG in the left and right side. Examining the muscle tension change in the splenius capitis muscle, a statistically significant within group difference was observed in both CTG and CSEG in the left and right side. In the between group comparison, a statistically significant difference was observed in the right side. The rest showed no statistically significant differences(p〉.05) (Table 4).

\section{DISCUSSION}

There are studies that have reported that the muscle strength and endurance of the cervical vertebra muscle among patients with cervical pain noticeably decrease $(14,15)$. Such changes in the strength of the cervical vertebra muscle and neck bone induces joint angle transformation of the cervical vertebra. The joint angle transformation of the cervical vertebra increases loading in the tissue around the neck $(16,17)$, and this phenomenon increases the muscle activity of the muscles around the neck bone(18). A forward head posture refers to a position that generally accompanies the flexion of the lower cervical vertebra and excessive extension of the upper cervical vertebra, which is frequently observed among office workers(19). This study attempts to provide fundamental clinical data for the treatment plan and rehabilitation of patients with cervical hypolordosis by comparing the cervical traction that is generally applied to patients with cervical hypolordosis and the cervical stretching exercise method that patients can perform by themselves.

Park et al. implemented cervical stretching exercise on 50 female subjects for four weeks and reported an improvement in cervical pain, muscle strength, flexibility, etc(20). Highland et al.'s study, which applied cervical exercise to $90 \mathrm{sub}-$ jects for eight weeks, reported a significant decrease in cervical pain(21). Moreover, Park, who had patients with cervical pain perform the MTT exercise program as a means of cervical exercise for six weeks, reported that improvements in muscle strength and pain reduction were observed(7). In our study, cervical pain also statistically significantly decreased in CSEG(p<.05). Incorrect posture and actions in daily life cause excessive use and shortening of the muscles on one side, which induces cervical pain. It is conjectured that cervical vertebra alignment improved as the cervical stretching exercise relaxed the stiffened muscle, correcting the balance of the muscle $(2,22)$.

Cervical lordosis can be found when observing the normal shape of the neck bone on the sagittal plane. However, as a result of using the head forward position frequently in cases such as using mobile electronic devices or computer monitors, cervical lordosis decreases and the muscle in the back of neck extends excessively, putting a lot of force and stress on the neck bone. This abnormal posture and the changes in the muscular contraction mechanism can induce headache and cervical pain by putting pressure on the facet joint of the cervical vertebra and disk. Muscles play the role of supporting the body in the opposite direction of stress to ensure momentum(16). Thuresson et al. compared a neutral posture and a $20^{\circ}$ forward flexion posture of the neck and reported that the $20^{\circ}$ forward flexion posture showed stronger muscle activity(23). Straker and Mekhora(2000) reported that a significant increase in the activity of the trapezius muscle and cervical erector spinae was observed as the neck tilted forward when using image display devices(24). Moreover, Yoo also reported that the activity of the upper trapezius muscle and splenius capitis muscle increased in a posture of forward cervical vertebra compared to a neutral posture when the subjects were watching digital multimedia broadcasting on their phones(25). In our study, the activity of the upper trapezius muscle and the splenius capitis muscle statistically significantly decreased in both CTG and CSEG. It is conjectured that the cervical traction method and the cervical stretching exercise decreased excessive stress on the cervical vertebra joint and muscle, impacting the cervical vertebra alignment positively. Consequently, the excessive tension in the upper trapezius muscle and splenius capitis muscle decreased $(2,22,25)$.

\section{CONCLUSION}

The purpose of this study is to provide fundamental clinical data for the treatment plan and rehabilitation of patients with cervical hypolordosis by comparing the cervical headache and muscle activity after cervical stretching exercise and cervical traction, which are generally applied to patients 
with cervical hypolordosis. The research subjects included 20 patients without gender division who were diagnosed with cervical hypolordosis. After applying cervical stretching exercise and cervical traction for six weeks, cervical headache and the activity of the muscles around the cervical vertebra(upper trapezius muscle, sternocleidomastoid muscle, splenius capitis muscle, and anterior temporal muscle) were investigated and the following results were obtained.

1. In a comparison of the within group intervention effects of the two groups, cervical pain statistically significantly decreased in the cervical stretching exercise group(p<.05).

2. According to the results of analyzing the change of muscle tension in the upper trapezius muscle, both the cervical traction group and showed a statistically significant withingroup difference in the left and right side(p<.05).

The results above confirm that cervical vertebra traction and cervical stretching exercise decrease the cervical headache and muscle activity of the upper trapezius muscle and splenius capitis muscle among subjects with cervical hypolordosis. Further studies will be required that investigate the impact on headache and neck muscle activity according to the cervical vertebra angle.

\section{REFERENCES}

1. Côté P, Cassidy JD, Carroll L. The Saskatchewan Health and Back Pain Survey. The prevalence of neck pain and related disability in Saskatchewan adults. Spine 1998; 23: 1689-1698.

2. Wang WT, Olson SL, Campbell AH, Hanten WP, Gleeson PB. Effectiveness of physical therapy for patients with neck pain: an individualized approach using a clinical decision-making algorithm. Am J Phys Med Rehabil 2003; 82(3): 203218.

3. Na BJ. The study of chiropractic technique on cervical disorder patients with military neck. Kyonggi University 2008.

4. Park HS. Effects of head posture on the rotational torque movement of mandible in patients with temporomandibular disorders. J Oral Med Pain 2000; 25(2): 173-189.
5. Hanten WP, Lucio RM, Russell JL, Brunt D. Assessment of total head excursion and resting head posture. Arch Phys Med Rehabil 1991; 72(11): 877-880.

6. Lee H, Lee BR, Park TG. Clinical studies on 50 cases of patient with cervical pain. J Kor acu moxi med soc 1999; 16(2): 69-82.

7. Park KD. The effect of MTT to neck pain patients cervical extension muscle strength and pain. Kor Alliance for Health, Physical education, Recreation, and Dance 2005; 44(5): 861-869.

8. Harris PR: Cervical traction : review of the literature and treatment guidelines. Phys Ther 1977; 57: 910-914.

9. Crue BL, Todd EM: The importance of flexion in cervical halter traction. Bull Los Angeles Neurol Soc 1965; 30: 95-98.

10. Brodie DJ, Burnett JV, Walker JM, Lydes-Reid D. Evaluation of low back

pain by patient questionnaires and therapist assessment. J Orthop Sports Phys Ther 1990; 11(11): 519-529.

11. Kim JT, Park SH. Effect of scapular stabilization exercise on EMG change of shoulder girdle and trunk muscle during archer's shooting. J Sport Leisure Studies 2009; 38: 1003-1010.

12. Jung WJ. Effects of a wallet in the back trouser pocket on paraspinal muscle activity in sitting. Yonsei University 2009.

13. Lee DH. The effects of Balance Exercise and Stretching Exercise on Forward Head Posture. Daegu University 2011.

14. Barton PM, and Hayes KC. Neck flexor muscle strength, efficiency, and relaxation times in normal subjects and subjects with unilateral neck pain and headache. Arch Phys Med Rehabil 1996; 77(7): 680-687.

15. Watson DH1, Trott PH. Cervical headache: an investigation of natural head posture and upper cervical flexor muscle performance. Cephalalgia. 1993; 13(4): 272-284.

16. Harms-Ringdahl K, Ekholm J. Intensity and character of pain and muscular activity levels elicited by maintained extreme flexion position of the lower cervical upper thoracic spine. Scand J Rehabil Med 1986; 18(3): 117-126.

17. Twomey L, Taylor J. Flexion creep deformation and hysteresis in the lumbar vertebral column. Spine 1982; 7(2): 116-122.

18. Schüldt K, Ekholm J, Harms-Ringdahl K, Németh G, Arborelius UP. Effects of changes in sitting work posture on static neck and shoulder muscle activity. Ergonomics 1986; 29(12): 1525-1537. 
19. Hanten WP1, Lucio RM, Russell JL, Brunt D. Assessment of total head excursion and resting head posture. Arch Phys Med Rehabil 1991; 72(11): 877-880.

20. Park HJ, Lee SN. The Effect on the Posture and Neck Pain in Head Backward Extension Exercise. Kor Soc Sport Leisure Studies 2002, 17; 223-231

21. Highland TR1, Dreisinger TE, Vie LL, Russell GS. Changes in isometric strength and range of motion of the isolated cervical spine after eight weeks of clinical rehabilitation. Spine 1992; 17: 77-82.

22. Ottawa. Mattresses for chronic back or neck pain: a review of the clinical effectiveness and guidelines. Canadian Agency for Drugs and Technologies in Health 2014.

23. Thuresson M, Ang B, Linder J, HarmsRingdahl K.Mechanical load and EMG activity in the neck induced by different head-worn equipment and neck postures. Int $\mathrm{J}$ Ind Ergon 2008; 35: 13-18.

24. Straker L, Mekhora K. An evaluation of visual display unit placement by electromyography, posture, discomfort and preference. Int $J$ Ind Ergon 2000; 26: 389-398.

25. Yoo CW. Eletromyographic activity of the neck and shoulder muscles while watching a DMB phone with the neck flexed. Yonsei University 2008. 